\title{
Dados de reprodução de Platyrrhinus lineatus em estudo de longo prazo no Estado do Rio de Janeiro (Mammalia, Chiroptera, Phyllostomidae)
}

\author{
Luciana M. Costa, Juliana C. Almeida \& Carlos E. L. Esbérard
}

Instituto de Biologia, Universidade Federal Rural do Estado do Rio de Janeiro, km 47 da antiga estrada Rio - São Paulo, Caixa Postal 74507, 23851-970 Seropédica, Rio de Janeiro, RJ, Brasil. (cesberard@ superig.com.br)

\begin{abstract}
Data on reproduction of Platyrrhinus lineatus in a long-term sampling study in the state of Rio de Janeiro (Mammalia, Chiroptera, Phyllostomidae). Based on a large bat sampling we analyzed the reproductive biology of Platyrrhinus lineatus (E. Geoffroy, 1810) in the Atlantic Forest, southeastern Brazil. The bats were captured with mist nets from 1989 and 2005 , in 27 of the 41 different localities sampled. The beginning of reproductive season was estimated 30 days before the date of capture of the first pregnant female. For determination of the end of the reproductive time we used the date of the capture of the last lactating female, added by 40 days. The total of 688 adult bats was analyzed, of which $58.3 \%$ were females. The largest numbers of captures was observed in the months of February, January and August. Males with descent testicles were observed in all of the months. Inactive females were observed in predominance in March and of May to August. Females with palpable fetuses were observed in all of the months except in April, June and July, with peak in August-October and January-February. Lactating females were netted from November to June, with peak in November and February. Females simultaneously pregnant and lactating were captured in all of the months except in July, with peak in April. The size of the reproductive season varied every year, from eight to 12 months, with an average of 10.6 months.
\end{abstract}

KEYWORDS. Reproductive season, seasonality, birth, lactation.

RESUMO. Baseado em uma grande amostragem de morcegos realizada na Mata Atlântica, Estado do Rio de Janeiro, sudeste do Brasil, nós analisamos a biologia reprodutiva de Platyrrhinus lineatus (E. Geoffroy, 1810). Os morcegos foram capturados com redes de neblina entre 1989 e 2005, em 27 das 41 diferentes localidades amostradas. O começo da época reprodutiva foi estimado 30 dias antes da data da captura da primeira fêmea grávida. Para determinação do término da época reprodutiva empregamos a data da captura da última fêmea lactante mais 40 dias. $\mathrm{O}$ total de 688 morcegos adultos foi analisado, sendo 58,3\% representados por fêmeas. O maior número de capturas foi observado nos meses de fevereiro, janeiro e agosto. Machos com testículos escrotados foram observados em todos os meses. Fêmeas inativas sexualmente foram observadas com predominância em março e de maio a agosto. Fêmeas com fetos palpáveis foram observadas em todos os meses, exceto em abril, junho e julho com pico em agosto-outubro e janeiro-fevereiro. Fêmeas lactantes foram capturadas de novembro a junho, com pico em novembro e fevereiro. Fêmeas simultaneamente grávidas e lactantes foram capturadas em todos os meses exceto em junho, com pico em abril. O tamanho da época reprodutiva variou a cada ano, compreendendo de 8 a 12 meses, com média de 10,6 meses.

PALAVRAS-CHAVE. Época reprodutiva, estacionalidade, nascimento, lactação.

Platyrrhinus lineatus (E. Geoffroy, 1810) ocorre da Colômbia ao Peru, Uruguai, até o norte da Argentina, Guiana Francesa e Suriname, incluindo o centro-oeste, nordeste, sudeste e sul do Brasil (Koopman, 1993).

Esta espécie, assim como seus congêneres, apresenta o conhecimento da biologia reprodutiva fragmentada. Os dados publicados são provenientes de coletas pontuais ou de pequeno número de capturas (WILsON, 1979). O gênero Platyrrhinus apresenta poliestria bimodal (WILSON, 1979). No Cerrado e Caatinga as fêmeas apresentam dois ciclos reprodutivos em cada época reprodutiva, com fêmeas grávidas na época seca e os primeiros partos ocorrendo na época das chuvas (WILLIG, 1985). Na Mata Atlântica, a época reprodutiva aparenta ser bem demarcada, com fêmeas grávidas presentes em agosto, setembro e outubro (Peracchi \& Albuquerque, 1971; Taddei, 1976; Teixeira \& Peracchi, 1996).

Este estudo apresenta os dados de reprodução proveniente de uma larga amostragem de morcegos realizada no Estado do Rio de Janeiro, sudeste do Brasil, no bioma da Mata Atlântica, que possibilitou uma análise detalhada da biologia reprodutiva de $P$. lineatus.

\section{MATERIAL E MÉTODOS}

Os morcegos foram capturados com redes de neblina armadas principalmente em trilhas já existentes e junto a árvores em frutificação, principalmente figueiras (Ficus spp.), entre 1989 e 2005 em 41 diferentes localidades do Estado do Rio de Janeiro (Fig. 1). A cada mês de duas a quatro noites de coleta foram realizadas e cada localidade foi amostrada pelo menos duas noites não consecutivas. Parte dos locais amostrados fazem parte de projeto de pesquisa que estuda as comunidades de morcegos em amostragens de longa duração e foram amostrados por mais de 10 noites. As localidades amostradas compreenderam desde florestas primárias a áreas urbanas, com altitudes variando de 0 a $1.100 \mathrm{~m}$. Os animais capturados foram identificados individualmente antes da soltura através de marcações temporárias com o uso de alicate tatuador ou com o uso de coleiras providas de cilindros coloridos (EsBÉrARD \& DAEMON, 1999), não sendo considerados os dados no caso de recapturas.

Para cada animal foram anotados os seguintes parâmetros: idade (distingüindo-se entre lactentes, jovens ou adultos através da ossificação das epífises) (ANTHONHY, 1988), cor do pelame, sexo e condição reprodutiva, 
inserindo os machos em testículos escrotados ou testículos abdominais, e as fêmeas em inativas sexualmente, com fetos palpáveis, com mamilos secretantes. Fêmeas simultaneamente grávidas e lactantes ou aquelas com mamilos desprovidos de pêlos e mamilos intumescidos com feto palpável foram agrupadas em fêmeas no segundo ciclo reprodutivo.

O início da época reprodutiva a cada ano foi estimado pela data da captura da primeira fêmea com feto palpável, considerando que só fetos com mais de 1/3 de gestação são detectáveis por este método (C. E. L. Esbérard, obs. pess.). Sendo a gestação nesta espécie esperada entre três e quatro meses (TADDEI, 1976), estimamos a concepção em pelo menos 30 dias antes. Para determinação do término da época reprodutiva empregamos a data da captura da última fêmea lactante, somando a esta 40 dias. Este valor corresponde a duração estimada da lactação para espécie com peso intermediário entre Carollia perspicillata (Linnaeus, 1758) e Artibeus jamaicensis Leach, 1821 (KunZ \& Hood, 2000).
No Estado do Rio de Janeiro a pluviosidade média anual varia de 1200 a $2200 \mathrm{~mm}$ por ano, não sendo observado nenhum mês com déficit hídrico (dados do Sistema Nacional de Meteorologia, normal de 1961 a 1990). A temperatura média anual varia de 18 a $24^{\circ} \mathrm{C}$. Os meses de junho e julho são os mais secos e a maior pluviosidade é observada em dezembro (Fig. 2).

Para testar o efeito da pluviosidade, fizemos regressão linear simples entre a pluviosidade média de cada mês usando a normal climatológica para a região central do Estado (1960-1990) com o total de capturas, o número de capturas de fêmeas grávidas e de lactantes.

\section{RESULTADOS}

Platyrrhinus lineatus foi capturado em 27 das 41 localidades já amostradas pelos autores no Estado do Rio de Janeiro (Tab. I). Um total de 688 exemplares adultos foi analisado (Fig. 3), com 58,29\% das capturadas representadas

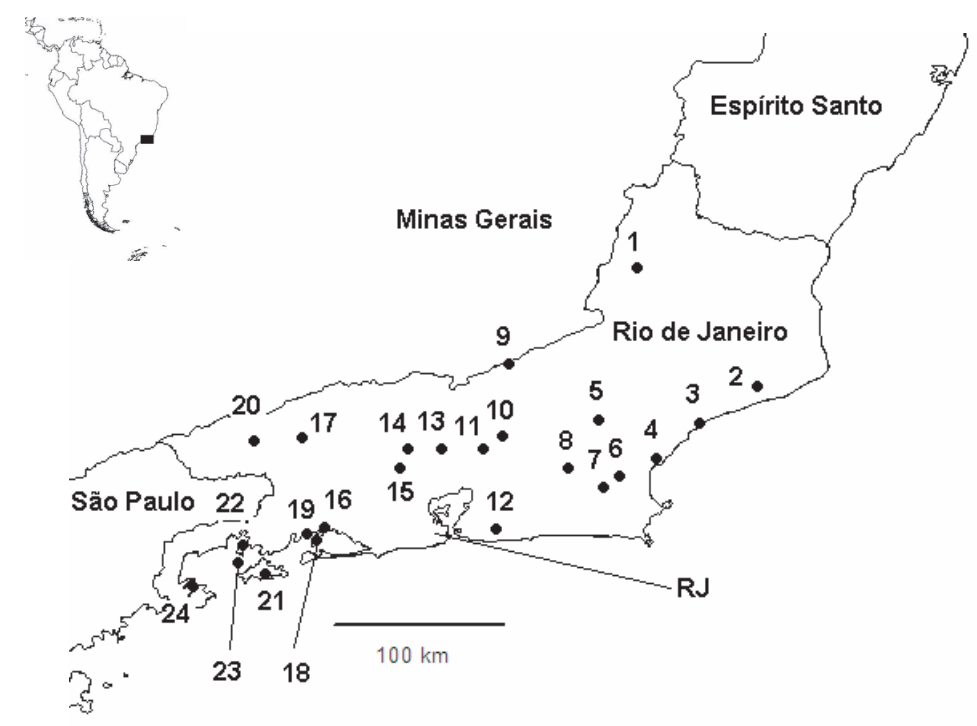

Fig. 1. Mapa com as localidades amostradas com a captura de Platyrrhinus lineatus no Estado do Rio de Janeiro (RJ, Cidade do Rio de Janeiro, onde a espécie foi capturada em 17 diferentes locais).

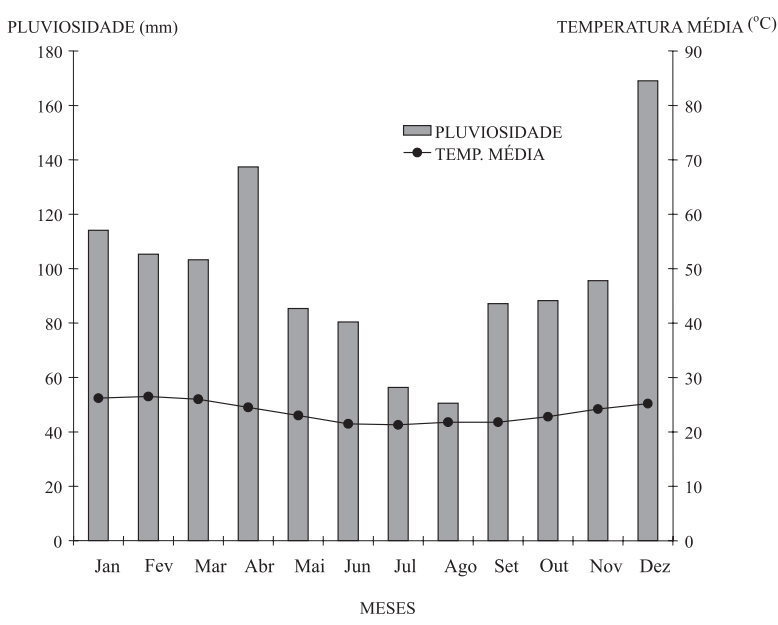

Fig. 2. Valores da normal climatológica (1960-1990) para a região central do Estado do Rio de Janeiro, segundo o Sistema Nacional de Meteorologia.

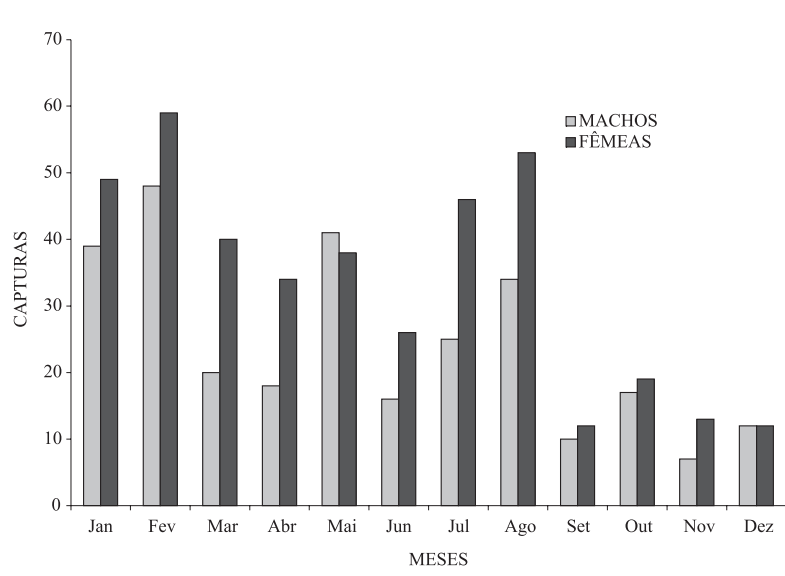

Fig. 3. Número de capturas de machos e fêmeas de Platyrrhinus lineatus capturados entre 1989 e 2005 em 25 localidades do Estado do Rio de Janeiro. 
por fêmeas. Fêmeas excederam os machos nas capturas em todos os meses. O maior número de capturas foi observado no mês de fevereiro $(15,55 \%)$ seguidos pelos meses de janeiro $(12,80 \%)$ e de agosto $(12,65 \%)$, no entanto, não foi obtida relação linear significativa do total de capturas com a pluviosidade $(r=0,062, p=0,847)$.

Machos com testículos escrotados foram observados em todos os meses. O número de machos com testículos abdominais superou o número de machos com testículos escrotados nos meses de fevereiro, junho, agosto e dezembro (Fig. 4).
Fêmeas inativas sexualmente foram observadas em todos os meses, com predominância (mais de $80 \%$ das capturas de fêmeas) em março e de maio a agosto. Fêmeas com fetos palpáveis foram observadas em todos os meses exceto em abril, junho e julho, com pico em agosto-outubro e janeiro-fevereiro. Fêmeas em estado de lactação foram capturadas de novembro a junho, com pico em novembro e fevereiro. Fêmeas simultaneamente grávidas e lactantes e na segunda gestação consecutiva foram capturadas em todos os meses exceto em julho

Tabela I. Localidades e coordenadas geográficas das localidades do Estado do Rio de Janeiro onde foram observadas capturas de Platyrrhinus lineatus entre 1989 e 2005 e total de capturas. (*) indicam locais de amostragens de médio ou longo prazo (mais de 10 noites de coleta).

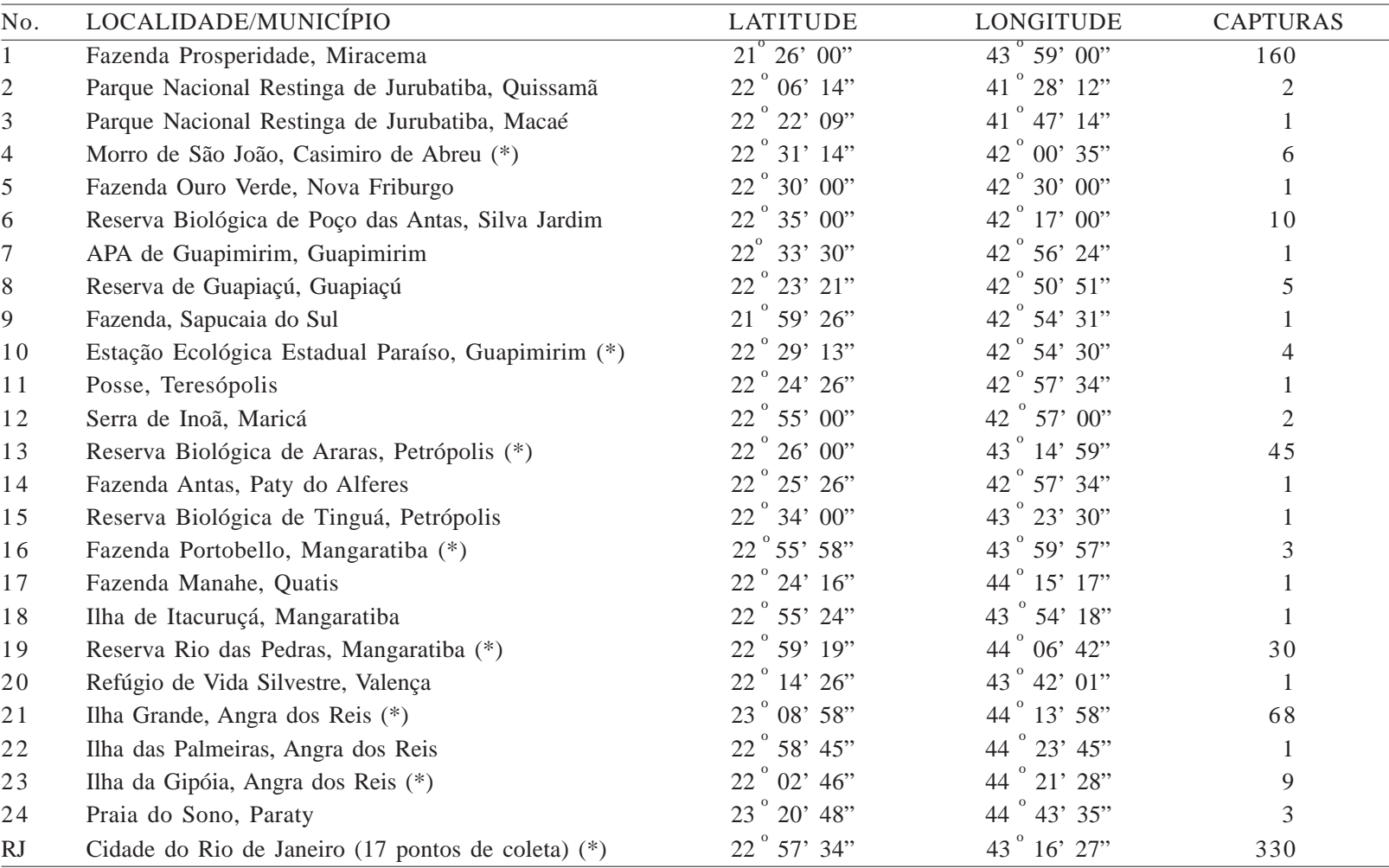

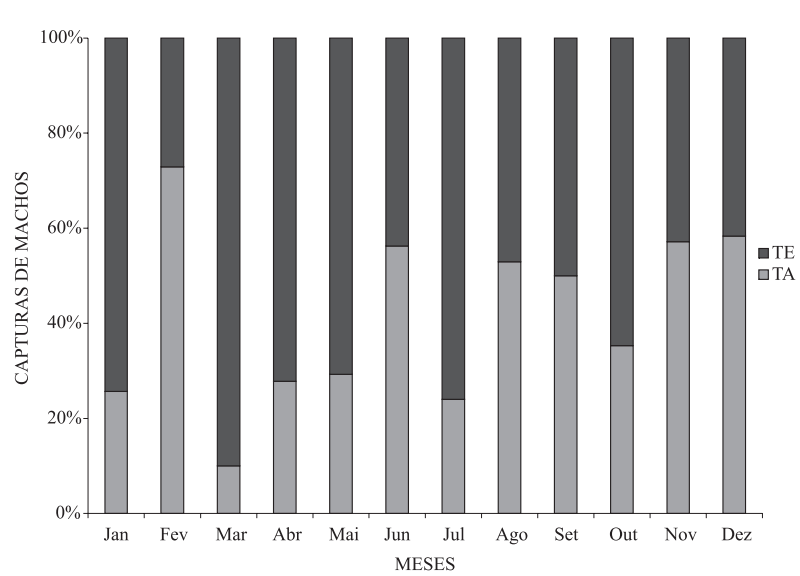

Fig. 4. Variação mensal no número de capturas de machos de Platyrrhinus lineatus com testículos abdominais e escrotados capturados entre 1989 e 2005 em 25 localidades do Estado do Rio de Janeiro (TA, testículos abdominais; TE, testículos escrotados).

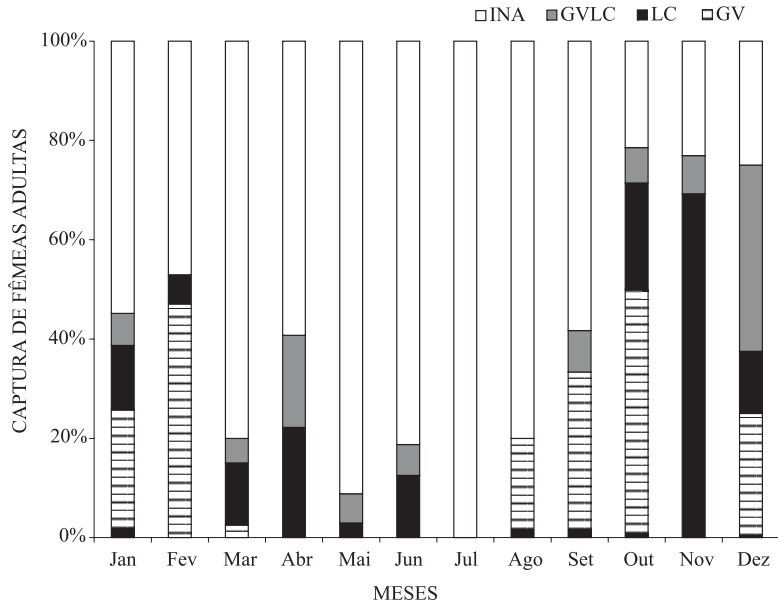

Fig. 5. Variação mensal de captura de fêmeas grávidas, lactantes e simultaneamente grávidas e lactantes em Platyrrhinus lineatus capturadas entre 1989 e 2005 em 25 localidades do Estado do Rio de Janeiro (INA, fêmeas inativas; GV, grávidas; GVLC, simultaneamente grávidas e lactentes e fêmeas com mamilos intumescidos e desprovidos de pêlos com feto palpável; LAC, lactentes). 
(Fig. 5). A ocorrência de fêmeas lactantes apresentou correspondência com a de fêmeas grávidas, ocorrendo dois ou três meses após. Foi obtida relação linear significante entre o total de capturas de fêmeas lactantes e a pluviosidade $(r=0,604, p=0,038)$, mas não com a de fêmeas grávidas ( $r=0,497, p=0,100)$ (Fig. 6).

Indivíduos subadultos estiveram presentes em todos os meses, exceto junho e julho. Indivíduos lactentes carregados pela reprodutora $(\mathrm{n}=3)$ foram observados em outubro, novembro e fevereiro e apresentaram comprimento do antebraço variando de 26,70 a $30,10 \mathrm{~mm}$ e peso variando de 7,0 a 9,0 g.

O tamanho do ciclo reprodutivo variou a cada ano, compreendendo de oito a 12 meses, com média de 10,58 \pm 1,24 meses, nos 13 anos em que foi possível estimar (Tab. II). Não foi obtida relação linear entre a duração estimada da época reprodutiva e o total capturado de fểmeas adultas $(\mathrm{r}=0,237, \mathrm{p}=0,471, \mathrm{n}=13)$.

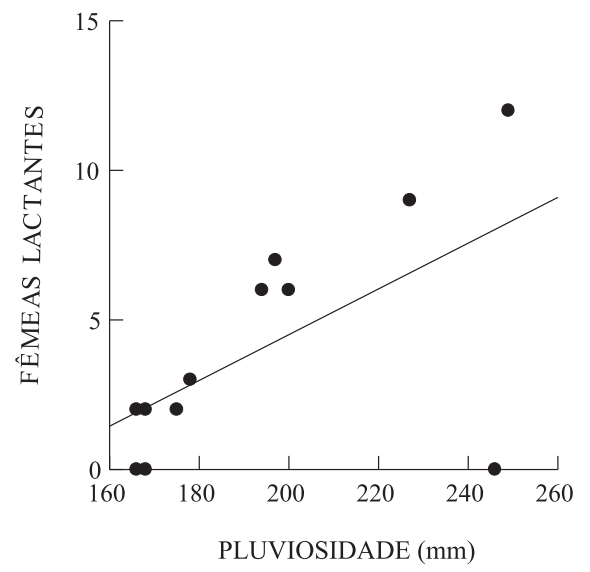

Fig. 6. Regressão linear entre o número de fêmeas lactantes de Platyrrhinus lineatus capturadas entre 1989 e 2005 em 25 localidades do Estado do Rio de Janeiro e os valores mensais de pluviosidade da normal climatológica (1960-1990).

Tabela II. Duração estimada da época reprodutiva de $P$. lineatus através da captura da primeira fêmea grávida e da última fêmea lactante a cada ano. $\mathrm{N}=$ número de fêmeas adultas capturadas a cada ano.

\begin{tabular}{|c|c|c|c|c|c|c|c|c|c|c|c|c|c|}
\hline \multicolumn{14}{|c|}{ MESES } \\
\hline ANOS & $\mathrm{N}$ & JUL & AGO & SET & OUT & NOV & DEZ & JAN & FEV & MAR & $\mathrm{ABR}$ & MAI & JUN \\
\hline 1989 & 16 & $*$ & $*$ & $*$ & $*$ & $*$ & $*$ & $*$ & $*$ & $*$ & $*$ & $*$ & $*$ \\
\hline 1990 & 52 & & $*$ & $*$ & $*$ & $*$ & $*$ & $*$ & $*$ & $*$ & $*$ & $*$ & \\
\hline 1991 & 30 & & & $*$ & $*$ & $*$ & $*$ & $*$ & $*$ & $*$ & $*$ & $*$ & $*$ \\
\hline 1992 & 12 & & $*$ & $*$ & $*$ & $*$ & $*$ & $*$ & $*$ & $*$ & $*$ & $*$ & \\
\hline 1993 & 23 & & & $*$ & $*$ & $*$ & $*$ & $*$ & $*$ & $*$ & $*$ & & \\
\hline 1994 & 8 & & & $*$ & $*$ & $*$ & $*$ & $*$ & $*$ & $*$ & $*$ & & \\
\hline 1995 & 17 & & $*$ & $*$ & $*$ & $*$ & $*$ & $*$ & $*$ & $*$ & $*$ & $*$ & $*$ \\
\hline 1996 & 36 & & & $*$ & $*$ & $*$ & $*$ & $*$ & $*$ & $*$ & $*$ & $*$ & $*$ \\
\hline 1997 & 26 & & & $*$ & $*$ & $*$ & $*$ & $*$ & $*$ & $*$ & $*$ & $*$ & $*$ \\
\hline 1998 & 23 & $*$ & $*$ & $*$ & $*$ & $*$ & $*$ & $*$ & $*$ & $*$ & $*$ & $*$ & $*$ \\
\hline 1999 & 36 & & $*$ & $*$ & $*$ & $*$ & $*$ & $*$ & $*$ & $*$ & $*$ & $*$ & \\
\hline 2000 & 40 & $*$ & $*$ & $*$ & $*$ & $*$ & $*$ & $*$ & $*$ & $*$ & $*$ & $*$ & $*$ \\
\hline 2001 & 39 & $*$ & $*$ & $*$ & $*$ & $*$ & $*$ & $*$ & $*$ & $*$ & $*$ & $*$ & $*$ \\
\hline
\end{tabular}

\section{DISCUSSÃO}

O número de fêmeas foi superior ao de machos em todos os meses, fato que pode ser explicado pelo hábito nesta espécie de formar haréns (NOWAK, 1995; Mello \& FERNANDEZ, 2000). Estes dados discordam do observado por TADDEI (1976) ao estudar a espécie em São Paulo, que obteve valor muito similar na proporção sexual. Machos com testículos escrotados foram capturados durante todo o ano. A dependência da atividade do ciclo reprodutivo masculino com o ciclo feminino foi observada entre espécies monogâmicas (TADDEI, 1976), mas em espécies poliéstricas, como $P$. lineatus, machos com testículos escrotados podem ser capturados a qualquer época (KRUTZSCh, 2000; RaCey \& ENTwistLe, 2000).

$\mathrm{O}$ maior número de capturas neste estudo foi observado no verão, que correspondem aos meses de alta pluviosidade, no entanto, não foi observação relação entre o total de capturas e a pluviosidade. Platyrrhinus lineatus é espécie frugívora (GARDNER, 1977), que pode ter sua proporção de captura dependente de alguns recursos nutricionais, como por exemplo, Cecropia pachystachya Trec. (Cecropiaceae) (PEDRo \& TADDEI, 2002). Dados provenientes do sudeste do Brasil indicam ser esta espécie tanto mais frequente na primeira metade da época chuvosa (PEDRo \& TADDEI, 2002) como na época mais seca (TADDEI, 1976). Sendo a captura desta espécie determinada por dois fatores principais, proximidade de refúgios e dos recursos nutricionais, amostragens pontuais ou com reduzido número de capturas, como as anteriormente citadas, podem ter tido maior influência de um ou dois destes fatores.

A reprodução em morcegos é sincronizada de modo que a lactação ocorra no período de maior disponibilidade de recursos, que ocorre no período de chuvas (JANZEN, 1967; Fleming et al., 1972; HeIDEMAN, 1995; ZoRTÉA, 2003), como observado neste trabalho. A atividade reprodutiva demanda altos gastos energéticos, principalmente durante a lactação (GitLemAN \& THOMPSON, 1988), sendo importante restringir esta atividade aos períodos onde há maior disponibilidade de recursos. Fêmeas grávidas de $P$. lineatus foram capturadas a partir de agosto, 
comprovando o início da estação reprodutiva em julho, com a ocorrência da cópula.

Espécies com longo período gestacional mostramse incapazes de reagir a rápidas mudanças ambientais e por isso restringem-se a reproduzir em épocas definidas, onde as condições são ótimas (RACEY \& ENSTwistLe, 2000). No entanto, a análise de várias épocas reprodutivas em $P$. lineatus demonstrou variações quanto ao início, duração e término, que podem estar associadas a fatores climáticos (como pluviosidade) e a disponibilidade de alimentos. Em anos em que a época reprodutiva é mais longa é provável uma maior proporção de nascimentos no segundo pico de nascimentos de $P$. lineatus ou probabilidade de sobrevivência maiores para os neonatos concebidos no segundo estro de cada fêmea.

Neste estudo foi observado que a época reprodutiva de $P$. lineatus dura em média 10 meses. A cada ano a duração estimada da época reprodutiva varia de 8 a 12 meses. A inexistência de relação entre o total de fêmeas capturadas a cada ano e a duração estimada da época reprodutiva corrobora a variação observada. Caso fosse significativa esta relação, os anos com menor duração poderiam ter sido subamostrados. A duração da época reprodutiva observada neste trabalho está de acordo com os dados obtidos para outras localidades, onde duas ninhadas consecutivas foram observadas em épocas reprodutivas que duram sete ou mais meses (WILSON, 1979). Este padrão é similar para outras espécies de Phyllostomidae estudadas a $23^{\circ}$ de latitude sul (Mello \& FernandeZ, 2000; MARINHO-FILHo, 2004).

Agradecimentos. Ao Instituto Estadual de Florestal, ao Instituto Brasileiro de Meio Ambiente, ao Centro de Primatologia do Rio de Janeiro, a Secretaria Municipal de Meio Ambiente do Município do Rio de Janeiro, a Secretaria de Agricultura e Pesca de Casimiro de Abreu, a Reserva Rio das Pedras e a Empresa de Pesquisas Agropecuária pela permissão para coletas e apoio concedidos. Lumilla M. S. Aguiar fez várias contribuições a este manuscrito. As coletas foram realizadas sob licença especial para coletas concedida pelo Instituto Brasileiro de Meio Ambiente (Processos 1755/89-SUPES/RJ/IBAMA e 4156/95-46 AC-SUPES/ DF/IBAMA). C. E. L. Esbérard recebeu uma bolsa do CNPq (processo 152910/2004-0).

\section{REFERÊNCIAS BIBLIOGRÁFICAS}

Anthony, E. L. P. 1988. Age determination in bats. In: Kunz, T. H. ed. Ecological and behavioral methods for the study of bats. Washington, Smithsonian Institution. p.47-58.

Esbérard, C. E. L. \& Daemon, C. 1999. Novo método para marcação de morcegos. Chiroptera Neotropical 5(1-2): 116-117.

Fleming, T. H.; Koper, E. T. \& Wilson, D. E. 1972. Three Central American communities: structure, reproductive cycles and movements patterns. Ecology 53(4):555-569.
Gardner, A. L. 1977. Feeding habits. In: Baker, R. J.; Carter, D C. \& Jones, J. K., Jr. eds. Biology of bats of the New World family Phyllostomidae. Part III. The Museum Texas Tech University Special Publications 13:293-350.

Gitleman, J. L. \& Thompson, S. D. 1988. Energy allocation in mammalian reproduction. American Zoologist 28:863-875.

Heideman, P. D. 1995. Synchrony and seasonality of reproduction in tropical bats. Symposia of the Zoological Society of London 67:151-165.

JANZEN, D. H. 1967. Synchronization of sexual reproduction of trees with the dry season in Central America. Evolution 21:620-637.

Koopman, K. F. 1993. Order Chiroptera. In: Wilson, D. E. \& Reeder, D. M. eds. Mammals species of the world: a taxonomic and geographic reference. 2. ed. Washinghton, Smithsonian Institution. p.137-232.

Krutzsch, P. 2000. Anatomy, physiology and ciclicity of the male reproductive tract. In: CRichton, E. G. \& Krutzsch, F. P. eds. Reproductive biology of bats. Boston, Academic Press. p.91-155.

Kunz, T. H. \& Hood, W. R. 2000. Parental care and postnatal growth in the Chiroptera. In: CRICHTON, E. G. \& KRUTZSCH, F. P. eds. Reproductive biology of bats. Boston, Academic Press. p.415-468.

Marinho-Filho, J. 2004. Notes on the reproduction of six phyllostomid bat species in southeastern Brazil. Chiroptera Neotropical 9(1-2): 173-175

Mello, M. A. R. \& Fernandez, F. A. S. 2000. Reproductive cycle of the bat Carollia perspicillata (Chiroptera: Phyllostomidae) in a fragment of the Brazilian Atlantic Coastal Forest. Zietschrifit Säugetierkunde 65:340-349.

NowAK, R. M. 1995. Walker's Mammals of the World. Baltimore, Johns Hopkins University Press. 1629p.

Pedro, W. A. \& Taddei, V. A. 2002. Temporal distribution of five bat species (Chiroptera, Phyllostomidae) from Panga Reserve, South-eastern Brazil. Revista Brasileira de Zoologia 19(3): 951-954.

Peracchi, A. L. \& Albuquerque, S. T. 1971. Lista provisória dos quirópteros dos estados do Rio de Janeiro e Guanabara, Brasil (Mammalia, Chiroptera). Revista Brasileira de Biologia 31: $405-413$

Racey, P. A. \& Entwistle, A. C. 2000. Life-history and reproductive strategies of bats. In: CRICHTON, E. G. \& KRUTZSCH, F. P. eds. Reproductive biology of bats. Boston, Academic Press. p.363-414.

TAdDeI, V. A. 1976. The reproduction of some Phyllostomidae (Chiroptera) from the northwestern region of the state of São Paulo. Boletim de Zoologia da Universidade de São Paulo 1: 313-330.

Teixeira, S. C. \& Peracchi, A. L. 1996. Morcegos do Parque Estadual da Serra da Tiririca, Rio de Janeiro, Brasil (Mammalia, Chiroptera). Revista Brasileira de Zoologia 13:61-66.

Willig, M. R. 1985. Reproductive patterns of bats from Caatingas and Cerrado biomes in northeastern Brazil Journal of Mammalogy 66:668-681.

WILSON, D. E. 1979. Reproductive patterns. In: BAKER, R. J.; CARTer, D. C. \& Jones, J. K., Jr. eds. Biology of bats of the New World family Phyllostomidae. Part III. The Museum Texas Tech University Special Publications 16:1-441.

ZorTÉA, M. 2003. Reproductive patterns and feeding habits of three nectarivorous bats (Phyllostomidae: Glossophaginae) from the brazilian Cerrado. Revista Brasileira de Biologia 63(1):159-168. 